\title{
NON-LINEAR DIFFERENTIAL EQUATIONS WITH TRANSCENDENTAL MEROMORPHIC SOLUTIONS
}

\author{
KATSUYA ISHIZAKI and YUEFEI WANG
}

(Received 22 November 1999; revised 17 July 2000)

Communicated by P. C. Fenton

\begin{abstract}
In this paper we treat two non-linear differential equations which come from complex dynamics theory. We give a complete classification of the equations when they possess transcendental meromorphic solutions.
\end{abstract}

2000 Mathematics subject classification: primary 34A20; secondary 30D35.

Keywords and phrases: meromorphic function, non-linear differential equation, Nevanlinna theory.

\section{Introduction and main results}

In this paper we consider the following equations with the assumption that they possess transcendental meromorphic solutions:

$$
\begin{aligned}
& \left(f^{\prime}\right)^{n}=q(z) P(f) P_{0}\left(f^{\prime}\right)(f-z)^{m}, \\
& \left(f^{\prime}\right)^{n}=q(z) e^{P_{1}(z)} P(f)(f-z)^{m},
\end{aligned}
$$

where $q(z)$ is a rational function, $P(z), P_{0}(z), P_{0}(0) \neq 0, P_{1}(z)$ are polynomials, and $m, n \in \mathbb{N}$. Here we use the word 'meromorphic' meaning meromorphic in the complex plane unless a domain is specified. The equations above have interesting properties of complex dynamics relating to the wandering domains of their solutions. The theory of complex dynamics is one of the active research areas in the complex analysis. An exposition can be found, for instance, in the recent books $[4,22]$ for

The first author is supported in part by a Grant-in-Aid for General Scientific Research from the Ministry of Education, Science and Culture 11640164 (Toda) and by a Grant from NIPPON Institute of Technology 0221 (1999).

The second author is supported by NSFC, JSPS and 973 Project.

(C) 2001 Australian Mathematical Society 0263-6115/2001 \$A2.00+0.00 
rational functions, [6] for transcendental functions. Recalling the theory of complex differential equations, it is possible that an equation possesses rational solutions and transcendental meromorphic solutions. The dynamical properties in the iteration of rational and transcendental solutions of differential equations have been studied in $[5,8,9,16,23]$. In [8], Bergweiler and Terglane considered the class $\mathscr{P}$ of all meromorphic solutions of the following differential equations:

$$
\begin{aligned}
f^{\prime} & =q(z)(f-z)^{2}, \\
f^{\prime} & =q(z)(f-\tau)(f-z), \\
\left(f^{\prime}\right)^{2} & =q(z)(f-\tau)(f-z)^{2}, \\
\left(f^{\prime}\right)^{2} & =q(z)(f-\tau)(f-\delta)(f-z)^{2},
\end{aligned}
$$

where $\tau$ and $\delta(\tau \neq \delta)$ are constants. They proved that no $f \in \mathscr{R}$ has wandering domains. Then Wang [23] discussed the wandering-domain problem for meromorphic solutions of the equations (1.1) and (1.2).

Bergweiler and Terglane mentioned in [8] the conditions of the existence of meromorphic solutions of the linear equation and the equations (1.3)-(1.6), citing [15]. In [9] they also gave references $[2,15]$ for the existence conditions of meromorphic solutions. We mention a reference, for instance [3], for the Riccati equation. However there are not many articles in which authors consider the existence theorems for the equations (1.1) and (1.2). The purpose of the present paper is to give complete classifications of the equations (1.1) and (1.2) when they possess transcendental meromorphic solutions. Our main results are as follows.

THEOREM 1.1. Suppose that the equation (1.1) has a transcendental meromorphic solution. Then (1.1) must be of one the equations of the form (1.3)-(1.6), or a linear equation of the form $f^{\prime}=q(z)(f-z)$.

THEOREM 1.2. Suppose that the equation (1.2) has a transcendental meromorphic solution. Then (1.2) must be one of the equations of the form

$$
\begin{aligned}
f^{\prime} & =q(z) e^{P_{1}(z)}(f-z), \\
f^{\prime} & =q(z) e^{P_{1}(z)}(f-z)^{2}, \\
f^{\prime} & =q(z) e^{P_{1}(z)}\left(f-\tau_{1}\right)(f-z), \\
\left(f^{\prime}\right)^{2} & =q(z) e^{P_{1}(z)}\left(f-\tau_{1}\right)(f-z)^{2}, \\
\left(f^{\prime}\right)^{2} & =q(z) e^{P_{1}(z)}\left(f-\tau_{1}\right)\left(f-\tau_{2}\right)(f-z)^{2},
\end{aligned}
$$

where $\tau_{1}$ and $\tau_{2}\left(\tau_{1} \neq \tau_{2}\right)$ are constants.

It is not intended to investigate further properties of the iteration of solutions in this note. We treat the equations (1.1) and (1.2) in Section 2 and Section 3 respectively. We 
use standard notations of the Nevanlinna theory, see for example [11, 19]. In particular, for a transcendental meromorphic function $f, S(r, f)$ denote any quantity that satisfies $S(r, f)=o(1) T(r, f)$ as $r \rightarrow \infty$, possibly outside a set of $r$ of finite linear measure. A meromorphic function $a$ is called small with respect to $f$ if $T(r, a)=S(r, f)$ holds. We employ the arguments from Malmquist-Yosida-Steinmetz type theorems, for example, $[15,17]$. In the remaining part of this section we state some lemmas below, which are needed later. First we recall the Mokhon'ko-Steinmetz type theorem, see for example, $[18,21]$.

LEMMA 1.3. Let $l$ be a positive integer. Suppose that an algebraic differential equation

$$
\Omega\left(z, f, f^{\prime}, \ldots, f^{(l)}\right)=0
$$

possesses an transcendental meromorphic solution $f(z)$. Let $\eta(z)$ be a meromorphic function small with respect to $f(z)$. If $\eta(z)$ does not satisfies the equation (1.12), then we have

$$
m\left(r, \frac{1}{f-\eta}\right)=S(r, f) .
$$

The following lemma is derived from the Nevanlinna second fundamental theory.

LEMMA 1.4. Let $f(z)$ be a transcendental meromorphic function, and let $a_{1}(z)$, $a_{2}(z)$ and $a_{3}(z)$ denote small functions with respect to $f(z)$ (possibly constant and $\infty$ ). Then we have

$$
T(r, f) \leq \sum_{j=1}^{3} \bar{N}\left(r, \frac{1}{f-a_{j}}\right)+S(r, f) .
$$

Zhu [25] generalized Hayman's inequality which is required in Section 3.

LEMMA 1.5. Let $f(z)$ be a transcendental meromorphic function and $\phi(z)$ be a small function with respect to $f(z)$. Then

$$
T(r, f) \leq 8 N\left(r, \frac{1}{f}\right)+8 N\left(r, \frac{1}{f^{\prime}-\phi}\right)+S(r, f) .
$$

Note that in (1.13), (1.14) and (1.15), we can write $O(\log r)$ in place of $S(r, f)$ if $f(z)$ is of finite order. Gol'dberg [10] showed that all transcendental meromorphic solutions of first order algebraic differential equations are of finite order, (see also Bank and Kaufman [1], Bergweiler [7] and Laine [17]). Hence when we treat solutions of (1.1) we may write $O(\log r)$ in place of $S(r, f)$. 


\section{Differential equation (1.1)}

At the beginning of this section we make the following remark. Under the assumption that (1.1) possesses a transcendental meromorphic solution we can write (1.1) as follows:

$$
\left(f^{\prime}\right)^{n}=q(z) P(f)\left(f^{\prime}-a\right)^{t}(f-z)^{m},
$$

where $a \in \mathbb{C} \backslash\{0\}, t \in \mathbb{N} \cup\{0\}$. In fact, in the case $P_{0}(z)$ is not a constant, we write

$$
P_{0}\left(f^{\prime}\right)=A_{0}\left(f^{\prime}-a_{1}\right)^{t_{1}} \cdots\left(f^{\prime}-a_{k}\right)^{t_{k}}, \quad \sum_{j=1}^{k} t_{j}=\operatorname{deg} P_{0},
$$

where $A_{0} \neq 0$ is a constant, and $a_{j}, j=1,2, \ldots, k$, are distinct constants and $t_{j}$, $j=1,2, \ldots, k$, are positive integers. It follows from $(1.1)$ that $a_{j} j=1, \ldots, k$ are Picard values of $f^{\prime}(z)$. This implies that $k$ must be 1 . Hence we may write $P_{0}\left(f^{\prime}\right)=\left(f^{\prime}-a\right)^{t}, a \in \mathbb{C} \backslash\{0\}, t \in \mathbb{N} \cup\{0\}$.

In the case $t=0$, by Bank and Kaufman [2], and Steinmetz [20], (2.1) is one of the equations of the form (1.3)-(1.6) or a linear equation when (2.1) has a transcendental meromorphic solution. In the sequel, we consider the case where $t$ is a positive integer. In the first part of this section we shall prove the main lemma (Lemma 2.1) and in the second part we shall treat separately the equations obtained in Lemma 2.1.

2.1. In this subsection, we prove the following lemma.

LEMMA 2.1. Suppose that $t>0$ in (2.1) and suppose that (2.1) possesses a transcendental meromorphic solution $f(z)$. Then $(2.1)$ is one of the equations of the form

$$
\begin{aligned}
& \left(f^{\prime}\right)^{n}=q(z)(f-z)^{n}\left(f^{\prime}-a\right)^{t}, \quad t<n, \\
& \left(f^{\prime}\right)^{n}=q(z)(f-\tau)^{n-2 t}(f-z)^{n}\left(f^{\prime}-a\right)^{t}, \quad 2 t \mid n .
\end{aligned}
$$

We need Lemma 2.2-Lemma 2.5 to prove Lemma 2.1. We write

$$
P(f)=A\left(f-\tau_{1}\right)^{r_{1}} \cdots\left(f-\tau_{k}\right)^{r_{k}}, \quad \sum_{j=1}^{k} r_{j}=p,
$$

where $A \neq 0$ is a constant, and $\tau_{j}, j=1,2, \ldots, k$, are distinct constants and $r_{j}$, $j=1,2, \ldots, k$, are positive integers.

We first observe the value distribution of poles of $f(z)$.

LEMMA 2.2. Suppose that $t>0, m \geq n$ in (2.1) and suppose that (2.1) possesses a transcendental meromorphic solution $f(z)$. Then $f(z)$ has infinitely many poles and $t<n$. 
PrOOF. First we consider the case $p \leq n$. We write (2.1) as

$$
\frac{\left(f^{\prime}\right)^{p}}{P(f)}\left(f^{\prime}\right)^{n-p}=q(z)\left(f^{\prime}-a\right)^{t}(f-z)^{m}
$$

Setting $f-z=g$, we have

$$
\left(g^{\prime}+1-a\right)^{t} g^{m}=\frac{1}{q(z)} \frac{\left(f^{\prime}\right)^{p}}{P(f)}\left(g^{\prime}+1\right)^{n-p} .
$$

Hence, in view of the Clunie lemma, see for example [11], and [17], we get

$$
\operatorname{tm}\left(r, f^{\prime}-a\right)=\operatorname{tm}\left(r, g^{\prime}+1-a\right) \leq m\left(r, \frac{\left(f^{\prime}\right)^{p}}{P(f)}\right)+S(r, g) \leq O(\log r) .
$$

Hence, $f^{\prime}(z)$ has infinitely many poles, and thus $f(z)$ has infinitely many poles.

Next we consider the case $p>n$. In the case there is no $r_{j}>n$ in (2.4), we can write $\left(f^{\prime}\right)^{n} / P(f)$ as:

$$
\begin{aligned}
\frac{\left(f^{\prime}\right)^{n}}{P(f)}= & \left(f^{\prime}\right)^{n}\left[\left(\frac{C_{11}}{f-\tau_{1}}+\frac{C_{12}}{\left(f-\tau_{1}\right)^{2}}+\cdots+\frac{C_{1 r_{1}}}{\left(f-\tau_{1}\right)^{r_{1}}}\right)\right. \\
& \left.+\cdots+\left(\frac{C_{k 1}}{f-\tau_{k}}+\frac{C_{k 2}}{\left(f-\tau_{k}\right)^{2}}+\cdots+\frac{C_{1 r_{k}}}{\left(f-\tau_{k}\right)^{r_{k}}}\right)\right] \\
= & \left(f^{\prime}\right)^{n} \sum_{h=1}^{k} \sum_{j=1}^{n} \frac{C_{h j}}{\left(f-\tau_{h}\right)^{j}}, \quad C_{h j}=0, j \geq r_{h}+1 \\
= & \left(\sum_{h=1}^{k} C_{h 1} \frac{f^{\prime}}{f-\tau_{h}}\right)\left(f^{\prime}\right)^{n-1}+\cdots+\sum_{h=1}^{k} C_{h n} \frac{\left(f^{\prime}\right)^{n}}{\left(f-\tau_{h}\right)^{n}},
\end{aligned}
$$

where $C_{h j}$ are constants. From the last equation it follows that $\left(f^{\prime}\right)^{n} / P(f)$ is a polynomial in $f^{\prime}$ and proximity functions of all coefficients are small order of growth. Hence in view of the Clunie theorem again, we obtain $m\left(r, f^{\prime}-a\right)=O(\log r)$ by the same argument as above. This gives that $f^{\prime}(z)$ has infinitely many poles, and hence $f(z)$ has infinitely many poles.

It remains to consider the case where $r_{j}>n$ for some $j$. We assume that $r_{1}>n$, $r_{2}>n, \ldots, r_{l}>n, l \leq k$ and $f(z)$ has only finitely many poles. Looking at the equation (2.1), $f(z)$ has only finitely many $\tau_{j}$-points for $j=1, \ldots, l$. By the Picard theorem, $l$ must be 1 . Hence we can write $f(z)$ as

$$
f(z)=R(z) e^{Q(z)}+\tau_{1},
$$

where $Q(z)$ is a polynomial and $R(z)$ is a rational function. It follows from (2.5) that the left-hand side of (2.1) is a polynomial in $e^{Q(z)}$ of degree $n$ with small coefficients. 
On the other hand, the right-hand side of (2.1) is a polynomial in $e^{Q(z)}$ of degrec $r_{1}+t+n s>n$ having small coefficients with respect to $e^{Q(z)}$, a contradiction. Therefore, $f(z)$ has infinitely many poles in the case $p>n$.

We show that $t<n$. In order to do this, we assume on the contrary that $t \geq n$. Since $f(z)$ has infinitely many poles, there exists a pole $z_{p}$ of $f(z)$ such that $q\left(z_{p}\right) \neq 0, \infty$. Write (2.1) as

$$
\frac{\left(f^{\prime}\right)^{n}}{\left(f^{\prime}-a\right)^{t}}=q(z) P(f)(f-z)^{m}
$$

From the assumption above, the left-hand side of (2.6) is analytic at $z_{p}$. However, the right-hand side of (2.6) has a pole at $z_{p}$, a contradiction.

We discuss the value distribution of fixed points of $f(z)$.

LEMMA 2.3. Suppose that $t>0$ in (2.1) and that (2.1) possesses a transcendental meromorphic solution $f(z)$. Then

$$
N\left(r, \frac{1}{f-z}\right)=T(r, f)+O(\log r) .
$$

Further, $m=n$ and $n-2 t-p \geq 0$.

ProOF. Obviously, $\eta(z)=z$ does not solve the equation (2.1). Thus by Lemma 1.3 and the first fundamental theorem due to Nevanlinna we get (2.7). Hence $f(z)$ has infinitely many fixed points. Let $z_{0}$ be a fixed point satisfying $q\left(z_{0}\right) \neq 0, \infty$. In a neighbourhood of $z_{0}$ we write

$$
f(z)-z=C\left(z-z_{0}\right)^{\lambda}+\cdots,
$$

where $C \neq 0$ and $\lambda \geq 1$ is an integer. Then

$$
f^{\prime}(z)=1+C \lambda\left(z-z_{0}\right)^{\lambda-1}+\cdots
$$

Substituting the above expansions into (2.1), we see that the right-hand side of (2.1) has a zero of order $\lambda m$ at $z_{0}$. Hence the left-hand side of (1.2) must have a zero at $z_{0}$, which is only attained for $\lambda=1$ and $C=-1$. The order of the zero of the left-hand side of (2.1) is $s n$ for some integer $s$, which gives that $m=s n$.

By the Valiron-Mokhon'ko theorem, see [17], and from (2.6), we have

$$
\begin{aligned}
\max (n, t) T\left(r, f^{\prime}\right)+O(\log r) & =T\left(r, \frac{\left(f^{\prime}\right)^{n}}{\left(f^{\prime}-a\right)^{t}}\right)=T\left(r, q(z) P(f)(f-z)^{m}\right) \\
& =(p+m) T(r, f)+O(\log r),
\end{aligned}
$$


that is,

$$
\max (n, t) T\left(r, f^{\prime}\right)=(p+m) T(r, f)+O(\log r)
$$

We have $T\left(r, f^{\prime}\right) \leq 2 T(r, f)+O(\log r)$. By Lemma 2.2, we have $t<n$. Using (2.8), we see that $p+m=p+s n<2 n$, which gives that $n=m,(s=1)$.

Now we have $t<n$ and $m=n$. By Lemma 2.2, $f$ has infinitely many poles. Hence we can take $z_{p}$ a pole of $f$ satisfying $q\left(z_{p}\right) \neq 0, \infty$. We denote by $\mu$ the order of pole at $z_{p}$. Write $(2.1)$ as

$$
\left(\frac{f^{\prime}}{f-z}\right)^{n}=q(z) P(f)\left(f^{\prime}-a\right)^{t} .
$$

The left-hand side of (2.9) has a pole of order $n$ at $z_{p}$, and the right-hand side admits a pole of order $p \mu+(\mu+1) t$. It gives that

$$
\mu=\frac{n-t}{p+t}
$$

Since $\mu$ is a positive integer, from (2.10) we get $n-2 t-p \geq 0$. Thus Lemma 2.3 is proved.

In view of Lemma 2.2 and Lemma 2.3, we need only treat the case $t<n$ and $m=n$. Next we consider the zeros of $P(f(z))$.

LEMMA 2.4. Suppose that (2.1) possesses a transcendental meromorphic solution $f(z)$. Then we have

$$
m\left(r, \frac{1}{f-\tau_{i}}\right)=O(\log r), \quad i=1,2, \ldots, k
$$

where $\tau_{i}, i=1,2, \ldots, k$, are given in (2.4).

ProOF. We fix $i$ and put $u=1 /\left(f-\tau_{i}\right)$ in (2.1). Then we get an algebraic differential equation in $u$

$$
\left(-u^{\prime}\right)^{n}=q(z) A \prod_{j \neq i}\left(1+\left(\tau_{j}-\tau_{i}\right) u\right)^{r_{j}} u^{n-2 t-p}\left(1+\left(\tau_{i}-z\right) u\right)^{n}\left(-u^{\prime}-a u^{2}\right)^{t}
$$

By Lemma 2.3, we have $n-2 t-p=n-2 t-\sum r_{j} \geq 0$, which gives that $2 n-r_{i}>n$. Hence $u^{2 n-r_{i}}$ is the term that attains the total degree of (2.12), and no other term attains the total degree of (2.12). Therefore, by the Clunie type theorem we obtain $m(r, u)=O(\log r)$. Thus we have proved (2.11). 
LEMMA 2.5. Suppose that (2.1) possesses a transcendental meromorphic solution $f(z)$. Then either $P(f)$ in $(2.1)$ is a constant or is of the form

$$
P(f)=A(f-\tau)^{n-2 t}, \quad 2 t \mid n .
$$

Proof. We assume that $P(f)$ is not a constant and use (2.4). By Lemma 2.3 we can set $m=n$ in (2.1). By Lemma $2.4, f(z)$ has infinitely many $\tau_{j}$ points for all $j=1,2, \ldots, k$. We fix $j$. Let $z_{j}$ be a $\tau_{j}$ point satisfying $q\left(z_{j}\right) \neq 0, \infty$. From (2.1), it is clear that $f^{\prime}\left(z_{j}\right)=0$. Hence we write $f(z)$ in a neighbourhood of $z_{j}$ as

$$
f(z)=\tau_{j}+B_{j}\left(z-z_{j}\right)^{v_{j}}+\cdots, \quad B_{j} \neq 0,
$$

where $v_{j} \geq 2$ is an integer. Substituting (2.14) into (2.1), we get

$$
\left(v_{j}-1\right) n=v_{j} r_{j}, \quad \text { that is, } \quad r_{j}=\left(1-1 / v_{j}\right) n .
$$

Hence from (2.15)

$$
p=\sum_{j=1}^{k} r_{j}=\sum_{j=1}^{k}\left(1-\frac{1}{v_{j}}\right) n=n k-n \sum_{j=1}^{k} \frac{1}{v_{j}} .
$$

Using $n-2 t-p \geq 0$ (see Lemma 2.3) we get

$$
k+\frac{2 t}{n} \leq 1+\sum_{j=1}^{k} \frac{1}{v_{j}} .
$$

We show that $k \leq 1$. In fact, we assume on the contrary that $k \geq 2$. Then by (2.17) and using $\sum_{j=1}^{k} 1 / v_{j} \leq k / 2$, we get $k+2 t / n \leq 1+k / 2$, and hence, $0<4 t / n \leq 2-k \leq 0$, which is absurd. Therefore, we consider the case $k=1$. From (2.15)

$$
(v-1) n=v p,
$$

where we write $\nu_{1}=v$ for the simplicity. Combining (2.10) and (2.18), we have

$$
\mu=1+\frac{n-2 v t}{v n-n+v t} .
$$

If $\mu \geq 2$, then $n-2 v t \geq(v-1) n+v t$, that is, $(2-v) n \geq 3 v t>0$, which gives a contradiction since $v \geq 2$. If $\mu=1$, then we have $n=2 v t$ and $p=n-2 t$ by (2.18), which gives the form (2.13).

Here we give the proof of Lemma 2.1 .

PROOF OF LEMMA 2.1. By Lemma 2.3, we may only consider the case $m=n$. By Lemma 2.5, either $P(f)$ must be a constant or $P(f)$ must be of the form (2.13). The former case yields an equation of the form (2.2) and the condition $t<n$ follows from Lemma 2.2. In the latter case, we see that (2.1) must be an equation of the form (2.3). 
2.2. In this subsection we treat the equations (2.2) and (2.3). We first show that the transcendental solutions of (2.2) and (2.3) satisfy a certain type second order differential equation.

LEMMA 2.6. Suppose that the following differential equation

$$
\left(f^{\prime}\right)^{n}=q(z) P(f)(f-z)^{n}\left(f^{\prime}-a\right)^{\prime}, \quad t<n
$$

possesses a transcendental meromorphic solution $f(z)$. Then $f(z)$ satisfies a second order differential equation with some rational function $r(z)$

$$
(\mu+1) \frac{f^{\prime}}{f-z}-\mu \frac{f^{\prime \prime}}{f^{\prime}-a}=r(z),
$$

where $\mu$ is given by (2.10).

PROOF. For the sake of brevity we denote by $F(z)$ the left-hand side of $(2.20)$. We show that $T(r, F)=O(\log r)$ which implies that $F(z)$ is a rational function. First we consider the counting function of $F(z)$. In the proof of Lemma 2.3, we obtained that almost all zeros of $f(z)-z$ are simple and from (2.19) they are also zeros of $f^{\prime}(z)$. Hence almost all poles of $f^{\prime}(z) /(f(z)-z)$ are only from poles of $f(z)$. We see from the proof of Lemma 2.2 that almost all poles of $f(z)$ are of order $\mu$ which is given by (2.10). Therefore only at poles of $f(z)$, the function $f^{\prime}(z) /(f(z)-z)$ has poles and their order is one having residue $-\mu$ with finitely many exceptions.

It is not difficult to see from (2.19) that $f^{\prime}(z)$ has only finitely many a-points. Hence only at poles of $f(z)$ the function $f^{\prime \prime}(z) /\left(f^{\prime}(z)-a\right)$ has poles and their order is one having residue $-\mu-1$ with finitely many exceptions. These properties implies that $F(z)$ has only finitely many poles, which gives $N(r, F)=O(\log r)$.

Secondly, we estimate the proximity function of $F(z)$. By Lemma 2.3 and by the theorem on logarithmic derivatives

$$
\begin{aligned}
m(r, F) & \leq m\left(r, \frac{f^{\prime}-1}{f-z}\right)+m\left(r, \frac{1}{f-z}\right)+m\left(r, \frac{f^{\prime \prime}}{f^{\prime}-a}\right)+O(\log r) \\
& =O(\log r) .
\end{aligned}
$$

Thus we have proved Lemma 2.6.

PROPOSITION 2.1. Differential equation (2.2) does not possess any transcendental meromorphic solutions.

PROOF. We assume that (2.2) possesses a transcendental meromorphic solution $f(z)$. Consider logarithmic derivatives of both sides of (2.2). Then we get

$$
n \frac{f^{\prime \prime}}{f^{\prime}}=\frac{q^{\prime}(z)}{q(z)}+n \frac{f^{\prime}-1}{f-z}+t \frac{f^{\prime \prime}}{f^{\prime}-a} \text {. }
$$


From (2.10), we have $\mu=(n-t) / t$. By Lemma 2.6, we see that for a rational function $r(z), f(z)$ satisfies

$$
\frac{n}{t} \frac{f^{\prime}}{f-z}-\frac{n-t}{t} \frac{f^{\prime \prime}}{f^{\prime}-a}=r(z) .
$$

Combining (2.21) and (2.22), we obtain the first order differential equation

$$
\left(C_{1}(z) f+C_{0}(z)\right) f^{\prime}+B_{1}(z) f+B_{0}(z)=0,
$$

with

$$
\begin{aligned}
& B_{1}(z)=\operatorname{ant} q(z) r(z), \quad B_{0}(z)=\operatorname{antz} q(z) r(z), \\
& C_{1}(z)=t(t-n) q(z) r(z)+(t-n) q^{\prime}(z), \\
& C_{0}(z)=\left(n^{2}(1-a)+n t(z r(z)-1)\right) q(z)+(t-n) z q^{\prime}(z) .
\end{aligned}
$$

By Lemma 2.2, $f(z)$ has infinitely many póles, and hence all rational functions $C_{0}, C_{1}$, $B_{0}$ and $B_{1}$ in (2.23) must vanish. Hence we have $r(z) \equiv 0, q^{\prime}(z) \equiv 0$ and $t=(1-a) n$. In what follows we write $q(z)$ as $q(\neq 0)$, since $q(z)$ is a constant. Using these results and (2.2), we have

$$
\left(\frac{a f^{\prime \prime}}{f^{\prime}-a}\right)^{n}=\left(\frac{f^{\prime}}{f-z}\right)^{n}=q\left(f^{\prime}-a\right)^{\prime},
$$

that is,

$$
\left(f^{\prime \prime}\right)^{n}=\frac{q}{a^{n}}\left(f^{\prime}-a\right)^{n+t} .
$$

Put $g=f^{\prime}-a$ in the equation above. Then $g(z)$ satisfies an equation of the form

$$
g^{\prime}=c g^{1+t / n}
$$

where $c$ is a constant. However, (2.24) has no transcendental meromorphic solution which is seen by quadrature, a contradiction.

In order to prove Proposition 2.2 we also need the following lemma.

LEMMA 2.7. There is no transcendental meromorphic function satisfying both equations

$$
\left(u^{\prime}\right)^{4}+q(z)(1+k(z) u)^{4}\left(a u^{2}+u^{\prime}\right)=0
$$

and

$$
-2 k(z) q(z)\left(u^{\prime}\right)^{2}+(m(z)+h(z) u) u^{\prime}+B(z)(1+k(z) u) u^{2}=0,
$$

where $q(z) \not \equiv 0, k(z) \not \equiv 0, m(z), h(z)$ and $B(z) \not \equiv 0$ are rational functions. 
PROOF. We assume that there exists a transcendental meromorphic function that satisfies both (2.25) and (2.26), and we shall derive a contradiction. To do this we eliminate $\left(u^{\prime}\right)^{2}$ by using (2.25) and (2.26) to obtain the equation of the form

$$
G(z, u) u^{\prime}+H(z, u)=0,
$$

where $G(z, u)$ and $H(z, u)$ are polynomials in $u$ whose coefficients are rational functions. By the Malmquist-Yosida theorem, see for instance Hille [14] and Laine [17], we have three possibilities:

(i) the equation (2.27) is a Riccati equation;

(ii) the equation (2.27) is a linear equation;

(iii) $G(z, u) \equiv 0$ and $H(z, u) \equiv 0$, that is, all coefficients of $G(z, u)$ and $H(z, u)$ vanish.

We need only treat the case (iii). In fact, it follows from (2.26) that almost all poles of $u(z)$ are double poles. Further, by the Clunie theorem we have $m(r, u)=S(r, u)$ $(=O(\log r)$ in the case $u(z)$ is of finite order). If $u(z)$ satisfies a Riccati equation, almost all poles of $u(z)$ are simple poles. If $u(z)$ satisfies a linear equation, $u(z)$ has only finitely many poles. Hence occurrences of cases (i) and (ii) are impossible.

We actually compute $G(z, u)$ and $H(z, u)$. For the sake of brevity, we omit the argument $z$, for instance, we write $q(z)=q$.

$$
\begin{aligned}
G(z, u)= & m^{3}+8 k^{3} q^{4}+\left(3 m^{2} h+32 k^{4} q^{4}\right) u+\left(3 m h^{2}+4 B k m q+48 k^{5} q^{4}\right) u^{2} \\
& +\left(h^{3}+4 B k^{2} m q+4 B k h q+32 k^{6} q^{4}\right) u^{3}+\left(4 B k^{2} h q+8 k^{7} q^{4}\right) u^{4} \equiv 0
\end{aligned}
$$

and

$$
\begin{aligned}
H(z, u)= & \left(B m^{2}+8 a k^{3} q^{4}\right) u^{2}+\left(B k m^{2}+2 B m h+32 a k^{4} q^{4}\right) u^{3} \\
& +\left(2 B k m h+B h^{2}+2 B^{2} k q+48 a k^{5} q^{4}\right) u^{4} \\
& +\left(B k h^{2}+4 B^{2} k^{2} q+32 a k^{6} q^{4}\right) u^{5}+\left(2 B^{2} k^{3} q+8 a k^{7} q^{4}\right) u^{6} \equiv 0
\end{aligned}
$$

From (2.28) and (2.29) we compute $a u^{2} G(z, u)-H(z, u)$ as

$$
\begin{aligned}
& \left(-B m^{2}+a m^{3}\right) u^{2}+\left(-B k m^{2}-2 B m h+3 a m^{2} h\right) u^{3} \\
& \quad+\left(-2 B k m h-B h^{2}+3 a m h^{2}-2 B^{2} k q+4 a B k m q\right) u^{4} \\
& \quad+\left(-B k h^{2}+a h^{3}-4 B^{2} k^{2} q+4 a B k^{2} m q+4 a B k h q\right) u^{5} \\
& \quad+\left(-2 B^{2} k^{3} q+4 a B k^{2} h q\right) u^{6} \equiv 0 .
\end{aligned}
$$

Observing the coefficients of $u^{6}$ and $u^{2}$ in (2.30), we get

$$
h=\frac{B k}{2 a} \quad \text { and } \quad m^{2}(-B+a m)=0 .
$$


According to the second relation in (2.31), we consider two cases. In the case $\boldsymbol{m}=$ $B / a$, from the coefficient of $u^{3}$ in (2.30), we have $-B^{3} k /\left(2 a^{2}\right)=0$, a contradiction. In the case $m=0$, the coefficients of $u^{4}$ and $u^{5}$ in (2.30) give

$$
-\frac{B k}{4 a^{2}}-2 q=0 \text { and }-\frac{B k}{8 a^{2}}-2 q=0,
$$

which is impossible since $B(z) k(z) \not \equiv 0$.

PROPOSITION 2.2. Differential equation (2.3) does not possess any transcendental meromorphic solutions.

Proof. We assume that (2.3) possesses a transcendental meromorphic solution $f(z)$. We note that almost all poles of $f(z)$ are simple poles. As in the proof of Proposition 2.1 , by Lemma 2.6 and (2.3), we have that $f(z)$ satisfies the following two differential equations:

$$
n \frac{f^{\prime \prime}}{f^{\prime}}=\frac{q^{\prime}(z)}{q(z)}+(n-2 t) \frac{f^{\prime}}{f-\tau}+n \frac{f^{\prime}-1}{f-z}+t \frac{f^{\prime \prime}}{f^{\prime}-a}
$$

and

$$
2 \frac{f^{\prime}}{f-z}-\frac{f^{\prime \prime}}{f^{\prime}-a}=r(z)
$$

where $r(z)$ is a rational function. Combining (2.32) and (2.33), we obtain the first order differential equation

$$
J_{2}(z)\left(f^{\prime}\right)^{2}+J_{1}(z, f) f^{\prime}+J_{0}(z, f)=0,
$$

with

$$
\begin{aligned}
J_{2}(z)= & q(z)(\tau-z)(n-2 t), \\
J_{1}(z)= & (f-\tau)\left(\left((t-n) q(z) r(z)-q^{\prime}(z)\right) f\right. \\
& \left.+q(z)((1-2 a) n+z r(z)(n-t))+z q^{\prime}(z)\right), \\
J_{0}(z)= & \operatorname{anq}(z) r(z)(f-z)(f-\tau) .
\end{aligned}
$$

Further, we put $u=1 /(f-\tau)$ in (2.34). Then we see that $u(z)$ satisfies the differential equation of the form

$$
\begin{gathered}
(n-2 t)(z-\tau) q(z)\left(u^{\prime}\right)^{2}+\left(h_{1}(z) u+h_{0}(z)\right) u^{\prime} \\
+\operatorname{anq}(z) r(z) u^{2}(1+(\tau-z) u)=0
\end{gathered}
$$

where $h_{0}(z)=q^{\prime}(z)+(n-t) q(z) r(z), h_{1}(z)=n q(z)(2 a-1)+(\tau-z) h_{0}(z)$ are rational functions. It is impossible that $r(z) \equiv 0$. In fact, if we assume the contrary, from (2.35) $u(z)$ has only finitely many poles, a contradiction. 
From (2.35), almost all poles of $u(z)$ are double poles. This implies that $f(z)$ has infinitely many $\tau$-points of order 2 . Hence we obtain $v=2$ and $n / 2 t=2$. Using this we may write (2.3) as

$$
\left(f^{\prime}\right)^{4}=\tilde{q}(z)(f-\tau)^{2}(f-z)^{4}\left(f^{\prime}-a\right),
$$

where $\tilde{q}(z)^{4}=q(z)$ is a rational function. From (2.36), $u(z)$ satisfies

$$
\left(u^{\prime}\right)^{4}=-\tilde{q}(z)(1+(\tau-z) u)^{4}\left(a u^{2}+u^{\prime}\right) .
$$

We write $\tilde{q}(z)$ simply as $q(z)$ and we consider (2.37) and also (2.35) with $n=4$, $t=1$, that is,

$$
\begin{aligned}
& q(z)(z-\tau)\left(u^{\prime}\right)^{2}+\left(3 r(z) q(z)+q^{\prime}(z)+(4 q(z)(2 a-1)\right. \\
& \left.\quad+(\tau-z)\left(3 r(z) q(z)+q^{\prime}(z)\right) u\right) u^{\prime}+4 a q(z) r(z)(1+(\tau-z) u) u^{2}=0 .
\end{aligned}
$$

However, by Lemma 2.7, equations (2.37) and (2.38) do not have any common transcendental meromorphic solutions, a contradiction.

PROOF OF THEOREM 1.1. Using Lemma 2.1, Proposition 2.1 and Proposition 2.2, we conclude that if $t>0$, then (2.1) has no transcendental meromorphic solution. Therefore, by the Steinmetz, Bank and Kaufman theorem for binomial equations, we obtain Theorem 1.1.

\section{Differential equation (1.2)}

In this section we are concerned with the differential equation (1.2) under the assumption that it possesses a transcendental meromorphic solution $f(z)$. We call $f(z)$ an 'admissible' solution, if $T\left(r, e^{P_{1}}\right)=S(r, f)$. By means of the He-Laine theorem [13] generalizing the Steinmetz theorem, if (1.2) has an admissible solution, then (1.2) reduces to one of the equations (1.7)-(1.11).

The natural question arises whether we would arrive at the same conclusion when we do not assume that $f(z)$ has the 'admissible' property.

Similarly as in Section 2, we divide this section into two parts. In the first part we investigate conditions which must be satisfied if (1.2) has a transcendental meromorphic solution (Lemma 3.2-Lemma 3.4). Combining these conditions we restrict forms of (1.2), which is a main purpose (Lemma 3.1) of the first part. In the second part we separately treat the equations that are obtained in the first part (Proposition 3.2Proposition 3.6), 
3.1. In the first part we prove the following lemma.

LEMMA 3.1. Suppose that the equation (1.2) has a transcendental meromorphic solution. Then (1.2) must be one of the following nine equations:

$$
\begin{aligned}
f^{\prime} & =q(z) e^{P_{1}(z)}(f-z)^{s}, \\
\left(f^{\prime}\right)^{n} & =q(z) e^{P_{1}(z)}(f-z)^{n+1}, \\
\left(f^{\prime}\right)^{n} & =q(z) e^{P_{1}(z)}\left(f-\tau_{1}\right)(f-z)^{n}, \\
\left(f^{\prime}\right)^{n} & =q(z) e^{P_{1}(z)}\left(f-\tau_{1}\right)^{n-1}(f-z)^{s n}, \\
\left(f^{\prime}\right)^{n} & =q(z) e^{P_{1}(z)}\left(f-\tau_{1}\right)^{n-1}(f-z)^{n+1}, \\
\left(f^{\prime}\right)^{n} & =q(z) e^{P_{1}(z)}\left(f-\tau_{1}\right)\left(f-\tau_{2}\right)^{n-1}(f-z)^{n}, \\
\left(f^{\prime}\right)^{2} & =q(z) e^{P_{1}(z)}\left(f-\tau_{1}\right)\left(f-\tau_{2}\right)(f-z)^{2 s}, \\
\left(f^{\prime}\right)^{2} & =q(z) e^{P_{1}(z)}\left(f-\tau_{1}\right)\left(f-\tau_{2}\right)(f-z), \\
\left(f^{\prime}\right)^{2} & =q(z) e^{P_{1}(z)}\left(f-\tau_{1}\right)\left(f-\tau_{2}\right)\left(f-\tau_{3}\right)(f-z),
\end{aligned}
$$

where $\tau_{j}, j=1,2,3$ are distinct constants, and s is a positive integer.

We state two lemmas relating poles and fixed points.

LEMMA 3.2. Suppose that (1.2) possesses a transcendental meromorphic solution $f(z)$ having infinitely many poles. Then almost all poles are of order

$$
\mu=\frac{n}{p+m-n},
$$

and

$$
n<p+m \leq 2 n,
$$

where $p=\operatorname{deg} P$.

PROOF. It follows from the hypothesis that there exists a pole $z_{0}$ such that $q\left(z_{0}\right) \neq$ $0, \infty$. We denote by $\mu$ the order of pole at $z_{0}$. The left-hand side of $(1.2)$ has a pole of order $n(\mu+1)$ at $z_{0}$, and the right-hand side admits a pole of order $(p+m) \mu$. Thus

$$
\mu=\frac{n}{p+m-n} \text {. }
$$

Since $\mu$ is a positive integer, we get (3.11) from (3.10).

LEMMA 3.3. Suppose that (1.2) possesses a transcendental meromorphic solution $f(z)$ having infinitely many fixed points. Then for a positive integer $s$

$$
m=n s .
$$


PROOF. Since we assume that $f(z)$ has infinitely many fixed points, there exists a fixed point $z_{0}$ satisfying $q\left(z_{0}\right) \neq 0, \infty$. In a neighbourhood of $z_{0}$ we write

$$
f(z)-z=C\left(z-z_{0}\right)^{\lambda}+\cdots,
$$

where $C \neq 0$ and $\lambda \geq 1$ is an integer. Then

$$
f^{\prime}(z)=1+C \lambda\left(z-z_{0}\right)^{\lambda-1}+\cdots .
$$

Substituting (3.13) and (3.14) into (1.2), we see that the right-hand side of (1.2) has a zero of order $\lambda m$ at $z_{0}$. Hence the left-hand side of (1.2) must have a zero at $z_{0}$, which is only attained by $\lambda=1$ and $C=-1$. Thus the order of the left-hand side of (1.2) is $s n$ for some integer $s$, the assertion follows.

Similarly as in Section 2, we write

$$
P(f)=A\left(f-\tau_{1}\right)^{r_{1}} \cdots\left(f-\tau_{k}\right)^{r_{k}}, \quad \sum_{j=1}^{k} r_{k}=p,
$$

where $A \neq 0$ is a constant, and $\tau_{j}, j=1,2, \ldots, k$ are distinct constants and $r_{j}$, $j=1,2, \ldots, k$ are positive integers.

LEMMA 3.4. Suppose that (1.2) possesses a transcendental meromorphic solution $f(z)$ and suppose that $f(z)$ has infinitely many $\tau_{j}$ points for an arbitrary fixed $j$. Then we have that almost all $\tau_{j}$ points are of multiplicity

$$
v_{j}=\frac{n}{n-r_{j}},
$$

where $v_{j}$ is a positive integer at least 2 .

PROOF. From the assumption, that is to say, $f(z)$ has infinitely many $\tau_{j}$ points, there exists a pole $z_{j}$ such that $q\left(z_{j}\right) \neq 0, \infty$. We denote by $v_{j}$ the order of pole at $z_{j}$. From (1.2), it is clear that $f^{\prime}\left(z_{j}\right)=0$. Hence we write $f(z)$ in a neighbourhood of $z_{j}$

$$
f(z)=\tau_{j}+B_{j}\left(z-z_{j}\right)^{\nu_{j}}+\cdots, \quad B_{j} \neq 0,
$$

where $v_{j} \geq 2$ is an integer. Substituting (3.17) into (1.2), we get

$$
\left(v_{j}-1\right) n=v_{j} r_{j}, \quad \text { namely, } \quad r_{j}=\left(1-\frac{1}{v_{j}}\right) n,
$$

which gives the assertion.

PROOF OF LEMMA 3.1. We divide the proof into four cases as follows:

I. $f(z)$ has infinitely many poles and infinitely many fixed points.

II. $f(z)$ has infinitely many poles and only finitely many fixed points.

III. $f(z)$ has only finitely many poles and infinitely many fixed points.

IV. $f(z)$ has only finitely many poles and only finitely many fixed points. 
Case I. $(f(z)$ has infinitely many poles and infinitely many fixed points.)

From (3.11), we see that $s$ is 1 or 2 in this case. In particular, in the case $s=2$ $(m=2 n), p$ must be 0 , say $P(f)$ is a constant. Thus (1.2) must be the equation of the form (1.8). Therefore, the case $s=1(m=n)$ is left to be considered. Further, we divide Case I into three cases below according to the number of the Picard values:

(I.i) $f(z)$ has infinitely many $\tau_{j}$ points for all $j$.

(I.ii) $f(z)$ has only finitely $\tau_{j}$ points for one $j$.

(I.iii) $f(z)$ has only finitely $\tau_{j}$ points for two $j$.

(I.i) By means of Lemma 3.4 and (3.18), we have $r_{j}=\left(1-1 / v_{j}\right) n$. Hence by Lemma 3.2

$$
(m+p=) n+\sum_{j=1}^{k}\left(1-\frac{1}{v_{j}}\right) n \leq 2 n,
$$

that is,

$$
k-1 \leq \sum_{j=1}^{k} \frac{1}{v_{j}} .
$$

Since $v_{j} \geq 2$ for all $j$, we see that the right-hand side of (3.19) is at most $k / 2$. This concludes that $k \leq 2$. Looking at (3.19) in the case $k=2$, we have $1 / v_{1}+1 / v_{2}=1$. This results in $\nu_{1}=\nu_{2}=2$, and hence $r_{1}=r_{2}=n / 2$. Hence (1.2) must be the equation of the form (1.11), (or (3.7)). Next we treat the case $k=1$. By Lemma 3.4 (or (3.18)), $r_{1}=n\left(v_{1}-1\right) / \nu_{1}$ which implies that (1.2) is an equation of the form (3.4) with $s=1$. In the case $k=0$ we arrived at (3.1) with $s=1$.

(I.ii) We may assume that $f(z)$ has only finitely $\tau_{1}$ points and has infinitely many $\tau_{j}$ points for $j=2, \ldots, k$. Similarly as in the case (i), we have

$$
(m+p=) n+r_{1}+\sum_{j=2}^{k}\left(1-\frac{1}{v_{j}}\right) n \leq 2 n, \quad \text { that is, } k-2+\frac{r_{1}}{n} \leq \sum_{j=2}^{k} \frac{1}{v_{j}} .
$$

The right-hand side is at most $(k-1) / 2$, which implies that $k+2 r_{1} / n \leq 3$, and hence $k \leq 2$.

First we treat the case $k=2$. We can set orders of poles and $\tau_{2}$ points $\mu$ and $\nu_{2}$ respectively with only finite exceptions by Lemma 3.2 and Lemma 3.4. Then in view of the Nevanlinna second fundamental theorem

$$
\begin{aligned}
m\left(r, \frac{1}{f-\tau_{2}}\right)+\frac{\nu_{2}-1}{\nu_{2}} N\left(r, \frac{1}{f-\tau_{2}}\right)+m(r, f) \\
\quad+\frac{\mu-1}{\mu} N(r, f)+m\left(r, \frac{1}{f-\tau_{1}}\right) \leq 2 T(r, f)+S(r, f) .
\end{aligned}
$$


From the assumption of (I.ii), $m\left(r, 1 / f-\tau_{1}\right)=T(r, f)+S(r, f)$. Thus we have

$$
T(r, f) \leq \frac{1}{\nu_{2}} N\left(r, \frac{1}{f-\tau_{j}}\right)+\frac{1}{\mu} N(r, f)+S(r, f) .
$$

If $r_{1}+r_{2}<n$ holds, then by Lemma 3.2, we have $\mu \geq 2$. It follows from (3.20) that $\mu=\nu_{2}=2$. Using $\nu_{2}=2$ and (3.18), we get $r_{2}=n / 2$. Further from (3.18), $n /\left(r_{1}+r_{2}\right)=n /\left(r_{1}+n / 2\right)=2$, namely, $r_{1}=0$, a contradiction. Hence $r_{1}+r_{2}=n$. By Lemma 3.4, we get $r_{1}=\left(v_{1}-1\right) n / \nu_{1}$ and $r_{2}=n / \nu_{1}$. Thus (1.2) must be an equation of the form (3.6).

In the case $k=1$, by Lemma 3.2, setting the order of poles $\mu$, we obtain $r_{1}=n / \mu$. Therefore, (1.2) must be an equation of the form (3.3) in this case.

(I.iii) We may assume that $f(z)$ has only finitely $\tau_{1}$ and $\tau_{2}$ points and has infinitely many $\tau_{j}$ points for $j=3, \ldots, k$. By the Nevanlinna second fundamental theorem, we see that $N\left(r, 1 / f^{\prime}\right)=S(r, f)$. Hence it follows from $(1.2)$ that $N(r, 1 /(f-z))=$ $S(r, f)$, which contradicts Lemma 1.4 .

Case II. $(f(z)$ has infinitely many poles and only finitely many fixed points.)

First we consider the case $k \geq 2$. We choose $\tau_{l}$ and $\tau_{i}$. We apply Lemma 1.4 to $\tau_{l}$, $\tau_{i}$ and $z$. From the assumption, say $f(z)$ has only finitely many fixed points, we get

$$
T(r, f) \leq \bar{N}\left(r, \frac{1}{f-\tau_{l}}\right)+\bar{N}\left(r, \frac{1}{f-\tau_{i}}\right)+S(r, f) .
$$

By Lemma 3.4, for any $j$ almost all $\tau_{j}$ points are of order $v_{j}$ which is given by (3.16). Hence in each case $f(z)$ has infinitely many $\tau_{j}$ points or $f(z)$ has only finitely many $\tau_{j}$ points, we have

$$
\bar{N}\left(r, \frac{1}{f-\tau_{j}}\right)=\frac{1}{\nu_{j}} N\left(r, \frac{1}{f-\tau_{j}}\right)+S(r, f) .
$$

It follows from (3.21) and (3.22) that

$$
\begin{aligned}
T(r, f) & \leq \frac{1}{v_{l}} N\left(r, \frac{1}{f-\tau_{l}}\right)+\frac{1}{v_{i}} N\left(r, \frac{1}{f-\tau_{i}}\right)+S(r, f) \\
& \leq \frac{1}{2}\left(N\left(r, \frac{1}{f-\tau_{l}}\right)+N\left(r, \frac{1}{f-\tau_{i}}\right)\right)+S(r, f) \\
& \leq T(r, f)+S(r, f) .
\end{aligned}
$$

Hence we see that $f(z)$ has infinitely many $\tau_{l}$ points and $\tau_{i}$ points, and that almost all those points are of order 2 . Thus for all $j$ we have $r_{j}=n / 2$ by Lemma 3.4, which implies $p=n k / 2$. In view of Lemma 3.2 we obtain $2 n \geq n k / 2+m$, namely, $4-2 m / n \geq k$. We conclude that $k$ is at most 3 . Therefore we divide Case II into four cases according to $k$ : 
(II.i) $k=3$.

(II.ii) $k=2$.

(II.iii) $k=1$.

(II.iv) $k=0$.

(II.i) As seen in the argument above, we have $v_{1}=v_{2}=v_{3}=n / 2$. Since we assume that $f(z)$ has infinitely many poles, we get by Lemma 3.2 that almost all poles are of order

$$
\mu:=\frac{n}{3 n / 2+m-n}=\frac{n}{n / 2+m},
$$

and we have $3 n / 2+m \leq 2 n$, namely, $m \leq n / 2$. Now we shall show that $m=n / 2$. To do this we suppose $m<n / 2$ and arrive at a contradiction. Using (3.23), we get $\mu \geq 2$. Applying Lemma 1.4 to $\tau_{1}, \infty$ and $z$, we have

$$
T(r, f) \leq \frac{1}{2} T(r, f)+\frac{1}{\mu} N(r, f)+S(r, f),
$$

hence,

$$
(\mu-2) T(r, f)=S(r, f) .
$$

Thus $\mu=2$, and hence $2=n /(n / 2+m)$, that is, $m=0$, a contradiction. Hence (1.2) must be the equation of the form (3.9).

(II.ii) In this case we also have $v_{1}=v_{2}=n / 2$. Hence, by Lemma 3.2, almost all poles are of order $\mu:=n / m$. It follows that $m \leq n$. If we assume $m<n$, then $\mu \geq 2$. Similarly to the case (II.i), by applying Lemma 1.4 , we obtain $\mu=2$. Thus $m=n / 2$, in this case $m<n$, and hence we arrive at the equation of the form (3.8). For the case $m=n$ we obtain (3.7) (or (1.11)).

(II.iii) Simply we write $r_{1}, \nu_{1}$, and $\tau_{1}$ as $r, v$ and $\tau$, respectively. By Lemma 1.4

$$
T(r, f) \leq \frac{1}{\nu} N\left(r, \frac{1}{f-\tau}\right)+\frac{1}{\mu} N(r, f)+S(r, f) .
$$

If $f(z)$ has only finitely many $\tau$ points, then $\mu=1$ and $m(r, f)=S(r, f)$ from (3.24). Hence we have $2 n=m+r$ by Lemma 3.2, which results in

$$
\left(f^{\prime}\right)^{n}=\tilde{q}(z) e^{P_{1}(z)}(f-\tau)^{2 n-m}(f-z)^{m} .
$$

We infer that $f(z)$ satisfies a Riccati equation. To show this, we set

$$
r_{0}(z)=\frac{f^{\prime}}{f-\tau}-\frac{f^{\prime}-1}{f-z} .
$$

Note that $r_{0}(z) \not \equiv 0$ otherwise $f(z)$ is a rational function. By the theorem of logarithmic derivatives we have $m\left(r, r_{0}\right)=S(r, f)$. The poles of $r_{0}(z)$ are $\tau$ points, fixed 
points or poles of $f(z)$. Since $f(z)$ possesses only finitely many $\tau$ points and fixed points, almost all poles of $r_{0}(z)$ are poles of $f(z)$. However, by the Laurent expansions of the right-hand side of (3.26) at poles of $f(z)$, poles of $f(z)$ are not poles of $r_{0}(z)$. This implies that $N\left(r, r_{0}\right)=S(r, f)$. Hence $r_{0}(z)$ is a small function with respect to $f(z)$, and hence $f(z)$ satisfies a Riccati equation given by (3.26), that is,

$$
f^{\prime}=\frac{r_{0}(z)}{\tau-z}(f-\tau)\left(f-z-\frac{1}{r_{0}(z)}\right) .
$$

In the case $z+1 / r_{0}(z)=\tau$, we see that

$$
g^{\prime}=-\frac{r_{0}(z)}{\tau-z}
$$

where $g=1 /(f-\tau)$. Since $r_{0}(z) /(\tau-z)$ is a small function with respect to $f(z)$ and also $g(z),(3.27)$ is impossible by the Hayman-Miles theorem [12], a contradiction. In the case $z+1 / r_{0}(z) \not \equiv \tau$, combining the Riccati equation above and (3.25), we get

$$
\frac{r_{0}(z)^{n}}{(\tau-z)^{n}}(f-\tau)^{m}\left(f-z-\frac{1}{r_{0}(z)}\right)^{n}=q(z) e^{P_{1}(z)}(f-\tau)^{n}(f-z)^{m},
$$

which gives that $N\left(r, 1 /\left(f-z-1 / r_{0}\right)\right)=S(r, f)$. We apply Lemma 1.4 to $\tau, z$ and $z+1 / r_{0}(z)$. Then we get $T(r, f)=S(r, f)$ which is absurd. Therefore $f(z)$ has infinitely many $\tau$ points. Then by Lemma 3.4

$$
2 \leq v=n /(n-r) .
$$

On the other hand, by Lemma 3.2

$$
\mu=n /(r+m-n) .
$$

It follows from (3.28) and (3.29) that $r=n(\nu-1) / \nu$ and $m-n+r=n / \mu$. Thus we see that $\nu \mid n$ and $\mu \mid n$. We can write $n=s \mu \nu$ for a nonzero integer $s$ (possibly negative). Here we get

$$
m=s \mu v-s \mu(v-1)+s v=s(\mu+v) .
$$

Hence we can represent (1.2) as

$$
\left(f^{\prime}\right)^{\mu \nu}=\tilde{q}(z) e^{P_{1}(z) / s}(f-\tau)^{2 \nu \mu-\mu-\nu}(f-z)^{\mu+\nu},
$$

where $\tilde{q}(z)=q(z)^{1 / s}$ is a rational function. From (3.24),

$$
T(r, f) \leq\left(\frac{1}{\mu}+\frac{1}{v}\right) T(r, f)+S(r, f),
$$

which implies that $1 \leq 1 / \mu+1 / \nu$, that is,

$$
(\mu-1)(v-1) \leq 1 \text {. }
$$


This gives $\mu=1$ in the case $\nu>2$, and $\mu=1$, or 2 in the case $\nu=2$. Therefore from (3.31) we obtain (3.5) and (3.3) with $n=2$.

(II.iv) By Lemma 3.2, $n /(m-n)=\mu$ is an integer. Thus we see that (1.2) must be the equation of the form (3.2) in this case.

Case III. $(f(z)$ has only finitely many poles and infinitely many fixed points.)

By Lemma 3.4, almost all $\tau_{j}$ points have the same multiplicity for fixed $j$. We apply the Nevanlinna second fundamental theorem

$$
\sum_{j=1}^{k}\left(m\left(r, \frac{1}{f-\tau_{j}}\right)+\frac{v_{j}-1}{\nu_{j}} N\left(r, \frac{1}{f-\tau_{j}}\right)\right)+m(r, f) \leq 2 T(r, f)+S(r, f) .
$$

From this inequality, we get

$$
(k-1) T(r, f) \leq \sum_{j=1}^{k} \frac{1}{v_{j}} N\left(r, \frac{1}{f-\tau_{j}}\right)+S(r, f) \leq \frac{k}{2} T(r, f)+S(r, f),
$$

which implies that $k-1 \leq k / 2$, and hence $k \leq 2$. In the case $k=2$, namely when the equality holds in (3.32), we see that $v_{1}=v_{2}=2$, and that $m\left(r, 1 /\left(f-\tau_{j}\right)\right)=S(r, f)$, for $j=1,2$. Thus by Lemma 3.4, $r_{1}=r_{2}=n / 2$. On the other hand, for any $k$ by Lemma 3.3, for a positive integer $s, m=s n$. Therefore (1.2) must be the equation of the form (3.7).

For the cases $k=1$, we first prove that $f(z)$ has infinitely many $\tau_{1}$ points. If we assume the contrary, we can write

$$
f(z)=\tau_{1}+R(z) e^{Q(z)},
$$

where $R(z)$ is a rational function and $Q(z)$ is a nonconstant entire function. Hence we obtain

$$
\left(\left(R^{\prime}(z)+R(z) Q^{\prime}(z)\right) e^{Q(z)}\right)^{n}=q(z) R(z)^{r_{1}} e^{r_{1} Q(z)+P_{1}(z)}(f(z)-z)^{m} .
$$

From (3.33) we have

$$
N\left(r, \frac{1}{f-z}\right)=\frac{n}{m} N\left(r, \frac{1}{R^{\prime}+R Q^{\prime}}\right)+O(\log r) \leq S(r, f) .
$$

It follows from (1.2) and from the assumption $\left(f(z)\right.$ has only finitely many $\tau_{1}$ points) that $N\left(r, 1 / f^{\prime}\right)=S(r, f)$. Putting $F(z)=f(z)-z$ and applying Lemma 1.5 to $F(z)$, we get

$$
\begin{aligned}
T(r, f) & =T(r, F)+O(\log r) \leq N\left(r, \frac{1}{F}\right)+N\left(r, \frac{1}{F^{\prime}+1}\right)+S(r, f) \\
& \leq N\left(r, \frac{1}{f-z}\right)+N\left(r, \frac{1}{f^{\prime}}\right)+S(r, f) \leq S(r, f),
\end{aligned}
$$


which is absurd. Hence by Lemma 3.4, $r_{1}=n\left(v_{1}-1\right) / v_{1}, v_{1}$ is a positive integer. Therefore, (1.2) must be the equation of the form (3.4).

In the case $k=0$, we immediately see that (1.2) must be the equation of the form (3.1) by Lemma 3.3.

Case IV. $(f(z)$ has only finitely many poles and only finitely many fixed points.) We can write $f(z)$ in this case as follows:

$$
f(z)=z+R(z) e^{Q(z)},
$$

where $R(z)$ is a rational function and $Q(z)$ is a nonconstant entire function. We assert that $P(f)$ is a constant. If we assume the contrary, by Lemma $3.4, f-\tau_{j}$ has infinitely many zeros almost all of which are of multiple order, or only finitely many zeros. This implies that $N\left(r, 1 /\left(f-\tau_{j}\right)\right) \leq \frac{1}{2} \bar{N}\left(r, 1 /\left(f-\tau_{j}\right)\right)+S(r, f)$. Then by Lemma 1.4 for the fixed $j$, we have

$$
\begin{aligned}
T(r, f) & \leq \bar{N}(r, f)+\bar{N}(r, 1 /(f-z))+\bar{N}\left(r, 1 /\left(f-\tau_{j}\right)\right)+S(r, f) \\
& \leq \frac{1}{2} T(r, f)+S(r, f),
\end{aligned}
$$

a contradiction. Therefore, $P(f)$ is a constant. Hence substituting (3.34) into (1.2), we get

$$
\left(1+\left(R^{\prime}(z)+R(z) Q^{\prime}(z)\right) e^{Q(z)}\right)^{n}=q(z) R(z)^{m} e^{m Q(z)+P_{1}(z)} .
$$

We assert that $R^{\prime}(z)+R(z) Q^{\prime}(z) \equiv 0$. If we assume the contrary, we see that $N\left(r,\left(R^{\prime}(z)+R(z) Q^{\prime}(z)\right) e^{Q(z)}\right)=S\left(r, e^{Q(z)}\right)$ and $N\left(r, 1 /\left(R^{\prime}(z)+R(z) Q^{\prime}(z)\right) e^{Q(z)}\right)=$ $S\left(r, e^{Q(z)}\right)$ since $R^{\prime}(z)+R(z) Q^{\prime}(z)$ is a small function with respect to $e^{Q(z)}$. Further it follows from (3.35) that $N\left(r, 1 /\left(1+\left(R^{\prime}(z)+R(z) Q^{\prime}(z)\right) e^{Q(z)}\right)\right)=O(\log r)$. Hence, by Lemma 1.4, we get $T\left(r, e^{Q(z)}\right) \leq S\left(r, e^{Q(z)}\right)$, a contradiction. Thus we have $R^{\prime}(z)+R(z) Q^{\prime}(z)=0$. Since $R(z)$ is a rational function, there is also no nonconstant entire function $Q(z)$ solving it, a contradiction.

We have considered Cases I-IV, thus Lemma 3.1 is proved.

3.2. In this subsection, we prove a sequence of lemmas and propositions from which Theorem 1.2 will follow.

PROPOSITION 3.1. Suppose that the equation (3.4) possesses a transcendental meromorphic solution. Then $n=1$, or $n=2$ and $s=1$, that is to say, (3.4) must be an equation of the form (3.1) or (3.3) (or (1.10)).

PrOOF. First we consider the case when $f(z)$ has infinitely many poles. Let $z_{0}$ be a pole of order $\mu$, which is not a zero of $q(z)$. By Lemma 3.2, we have $\mu s n=\mu+n$, 
which gives $n=1, s=2$ or $n=2, s=1$. Secondly we consider the case that $f(z)$ has only finitely many poles.

By Lemma 3.4, almost all $\tau_{1}$ points are of multiplicity $n$. This implies that

$$
\bar{N}\left(r, \frac{1}{f-\tau_{1}}\right)=\frac{1}{n} N\left(r, \frac{1}{f-\tau_{1}}\right)+S(r, f) .
$$

By the Nevanlinna first fundamental theorem and the theorem on the logarithmic derivatives,

$$
\begin{aligned}
T(r, f) & -N\left(r, \frac{1}{f-\tau_{1}}\right) \\
= & m\left(r, \frac{1}{f-\tau_{1}}\right)+O(1) \leq m\left(r, \frac{f^{\prime}}{f-\tau_{1}}\right)+m\left(r, \frac{1}{f^{\prime}}\right)+O(1) \\
& \leq m\left(r, \frac{1}{f^{\prime}}\right)+S(r, f)=T\left(r, f^{\prime}\right)-N\left(r, \frac{1}{f^{\prime}}\right)+S(r, f),
\end{aligned}
$$

that is,

$$
N\left(r, \frac{1}{f^{\prime}}\right) \leq N\left(r, \frac{1}{f-\tau_{1}}\right)+T\left(r, f^{\prime}\right)-T(r, f)+S(r, f) .
$$

From the assumption $N(r, f)=S(r, f)$, and hence $N\left(r, f^{\prime}\right)=S(r, f)$. Thus, by the theorem on the logarithmic derivatives, we get $T\left(r, f^{\prime}\right)-T(r, f) \leq S(r, f)$. Hence we see that

$$
N\left(r, \frac{1}{f^{\prime}}\right) \leq N\left(r, \frac{1}{f-\tau_{1}}\right)+S(r, f) .
$$

On the other hand, by (3.4),

$$
\frac{n-1}{n} N\left(r, \frac{1}{f-\tau_{1}}\right)+s N\left(r, \frac{1}{f-z}\right)=N\left(r, \frac{1}{f^{\prime}}\right)+S(r, f) .
$$

Hence we obtain

$$
N\left(r, \frac{1}{f-z}\right) \leq \frac{1}{n s} N\left(r, \frac{1}{f-\tau_{1}}\right) .
$$

Then by Lemma 1.4, (3.36) and (3.37),

$$
\begin{aligned}
T(r, f) & \leq \bar{N}(r, f)+\bar{N}\left(r, \frac{1}{f-\tau_{1}}\right)+N\left(r, \frac{1}{f-z}\right)+S(r, f) \\
& \leq\left(\frac{1}{n}+\frac{1}{n s}\right) N\left(r, \frac{1}{f-\tau_{1}}\right)+S(r, f) .
\end{aligned}
$$

We conclude that $s+1 \geq n s$, which implies $n=1$, or $n=2$ and $s=1$. 
PROPOSITION 3.2. Suppose that the equation (3.7) possesses a transcendental meromorphic solution. Then $s=1$, that is to say, (3.7) must be an equation of the form (1.11).

PROOF. If $f(z)$ has infinitely many poles, then by Lemma 3.2 we get $s=1$.

Now suppose that $f(z)$ has only finitely many poles. It follows from Lemma 3.4 that almost all $\tau_{j}$-points of $f(z)$ are of multiplicity 2 for $j=1,2$, which implies

$$
\bar{N}\left(r, \frac{1}{f-\tau_{j}}\right)=\frac{1}{2} N\left(r, \frac{1}{f-\tau_{j}}\right)+S(r, f), \quad j=1,2 .
$$

Similarly as in the proof of Proposition 3.1, we have

$$
\begin{aligned}
2 T(r, f) & -N\left(r, \frac{1}{f-\tau_{1}}\right)-N\left(r, \frac{1}{f-\tau_{2}}\right) \\
= & m\left(r, \frac{1}{f-\tau_{1}}\right)+m\left(r, \frac{1}{f-\tau_{2}}\right)+O(1)=m\left(r, \frac{1}{f-\tau_{1}}+\frac{1}{f-\tau_{2}}\right)+S(r, f) \\
& \leq m\left(r, \frac{f^{\prime}}{f-\tau_{1}}\right)+m\left(r, \frac{f^{\prime}}{f-\tau_{2}}\right)+m\left(r, \frac{1}{f^{\prime}}\right) \leq m\left(r, \frac{1}{f^{\prime}}\right)+S(r, f) \\
& =T\left(r, f^{\prime}\right)-N\left(r, \frac{1}{f^{\prime}}\right)+S(r, f),
\end{aligned}
$$

that is,

$$
N\left(r, \frac{1}{f^{\prime}}\right) \leq N\left(r, \frac{1}{f-\tau_{1}}\right)+N\left(r, \frac{1}{f-\tau_{2}}\right)+T\left(r, f^{\prime}\right)-2 T(r, f)+S(r, f) .
$$

Using $T\left(r, f^{\prime}\right)-T(r, f) \leq S(r, f)$, we get

$$
N\left(r, \frac{1}{f^{\prime}}\right) \leq N\left(r, \frac{1}{f-\tau_{1}}\right)+N\left(r, \frac{1}{f-\tau_{2}}\right)-T(r, f)+S(r, f) .
$$

On the other hand, from (3.7),

$$
\frac{1}{2}\left(N\left(r, \frac{1}{f-\tau_{1}}\right)+N\left(r, \frac{1}{f-\tau_{2}}\right)\right)+s N\left(r, \frac{1}{f-z}\right)=N\left(r, \frac{1}{f^{\prime}}\right) .
$$

Hence we obtain

$$
\begin{aligned}
\frac{1}{s} T(r, f)+N\left(r, \frac{1}{f-z}\right) & \leq \frac{1}{2 s}\left(N\left(r, \frac{1}{f-\tau_{1}}\right)+N\left(r, \frac{1}{f-\tau_{2}}\right)\right)+S(r, f) \\
& \leq \frac{1}{s} T(r, f)+S(r, f) .
\end{aligned}
$$


This implies that $N(r, 1 /(f-z))=S(r, f)$. Applying Lemma 1.4 to $\tau_{1}, \infty$ and $z$, we get

$$
\begin{aligned}
T(r, f) & \leq \bar{N}(r, f)+\bar{N}\left(r, \frac{1}{f-\tau_{1}}\right)+\bar{N}\left(r, \frac{1}{f-z}\right)+S(r, f) \\
& \leq \bar{N}\left(r, \frac{1}{f-\tau_{1}}\right)+S(r, f) \leq \frac{1}{2} T(r, f)+S(r, f),
\end{aligned}
$$

which is absurd. Hence this case does not occur. This completes the proof.

PROPOSITION 3.3. $\quad$ (i) Suppose that the equation (3.3) possesses a transcendental meromorphic solution. Then $n \leq 2$, that is to say, (3.3) must be an equation of the form (1.9) or (1.10).

(ii) Suppose that the equation (3.6) possesses a transcendental meromorphic solution. Then $n \leq 2$, that is to say, (3.6) must be an equation of the form (1.10) or (1.11).

To prove Proposition 3.3, we need the lemma below.

LEMMA 3.5. Let $a(z)$ be a nonconstant rational function. Suppose that the differential equation

$$
\left(f^{\prime}\right)^{n}=q(z) e^{P_{1}(z)}\left(f-\tau_{1}\right)(f-a(z))^{n},
$$

possesses a transcendental meromorphic solution $f(z)$. Then $n \leq 2$.

PROOF. At the beginning of the proof we remark that Lemma 3.2-Lemma 3.4 still hold for the equation in which we put $a(z)$ in place of $z$ in (1.2). Assume that $n \geq 3$. By Lemma 3.4, $f(z)$ has only finitely many $\tau_{1}$ points, and hence

$$
T(r, f)=m\left(r, \frac{1}{f-\tau_{1}}\right)+S(r, f) .
$$

In view of the Nevanlinna second fundamental theorem,

$$
\begin{aligned}
& m(r, f)+N(r, f)-\bar{N}(r, f)+m\left(r, \frac{1}{f-\tau_{1}}\right)+N\left(r, \frac{1}{f^{\prime}}\right) \\
& \leq 2 T(r, f)+S(r, f) .
\end{aligned}
$$

Combining (3.41) and (3.40), we get

$$
N\left(r, \frac{1}{f^{\prime}}\right) \leq \bar{N}(r, f)+S(r, f) .
$$


From (3.39),

that is,

$$
\begin{aligned}
n N\left(r, \frac{1}{f^{\prime}}\right) & =N\left(r, \frac{1}{f-\tau_{1}}\right)+n N\left(r, \frac{1}{f-a}\right)+S(r, f) \\
& =n N\left(r, \frac{1}{f-a}\right)+S(r, f),
\end{aligned}
$$

$$
N\left(r, \frac{1}{f^{\prime}}\right)=N\left(r, \frac{1}{f-a}\right)+S(r, f) .
$$

Here we consider the order of poles of $f(z)$. By Lemma 3.2, if $f(z)$ has infinitely many poles, then almost all poles are of order $n$. It gives that

$$
\bar{N}(r, f)=\frac{1}{n} N(r, f)+S(r, f) .
$$

It follows from (3.42), (3.43) and (3.44) that

$$
N\left(r, \frac{1}{f-a}\right) \leq \frac{1}{n} N(r, f)+S(r, f) .
$$

In view of Lemma 1.4, we have

$$
m\left(r, \frac{1}{f-a}\right)+m\left(r, \frac{1}{f-\tau_{1}}\right)+N(r, f)-\bar{N}(r, f) \leq 2 T(r, f)+S(r, f) .
$$

Using (3.46) and (3.40), we obtain

$$
T(r, f)-N\left(r, \frac{1}{f-a}\right)+T(r, f)+N(r, f)-\bar{N}(r, f) \leq 2 T(r, f)+S(r, f),
$$

and hence by (3.44) and (3.45),

$$
N(r, f) \leq N\left(r, \frac{1}{f-a}\right)+\bar{N}(r, f)+S(r, f) \leq \frac{2}{n} N(r, f)+S(r, f) .
$$

This implies that $N(r, f)=S(r, f)$ since $n \geq 3$. Applying Lemma 1.4 again to $\infty$, $\tau_{1}$ and $a(z)$, and using (3.45), we obtain

$$
\begin{aligned}
T(r, f) & \leq \bar{N}(r, f)+\bar{N}\left(r, \frac{1}{f-\tau_{1}}\right)+\bar{N}\left(r, \frac{1}{f-a}\right)+S(r, f) \\
& \leq \frac{1}{n} N(r, f)+S(r, f) .
\end{aligned}
$$

This gives a contradiction. Thus we have proved Lemma 3.5. 
PROOF OF PROPOSITION 3.3. We see that (i) is confirmed directly by Lemma 3.5. For the proof of (ii), we set $g=1 /\left(f-\tau_{1}\right)$ in (3.6). Then we have

$$
\left(g^{\prime}\right)^{n}=(-1)^{n}\left(\tau_{1}-\tau_{2}\right)\left(\tau_{1}-z\right) q(z) e^{P_{1}(z)}\left(g-\frac{1}{\tau_{2}-\tau_{1}}\right)\left(g-\frac{1}{z-\tau_{1}}\right)^{n} .
$$

By Lemma 3.5, we obtain $n \leq 2$. We have thus proved Proposition 3.3.

PROPOSITION 3.4. $\quad$ (i) Suppose that the equation (3.2) possesses a transcendental meromorphic solution. Then $n=1$, that is to say, (3.2) must be an equation of the form (1.8).

(ii) Suppose that the equation (3.5) possesses a transcendental meromorphic solution. Then $n=1$, that is to say, (3.5) must be an equation of the form (1.8).

PROOF. We first prove (ii). Suppose $n \geq 2$ and that (3.5) possesses a transcendental meromorphic solution $f(z)$. We put $f=\tau_{1}+1 / g$ in (3.5). Then we see that $g$ satisfies

$$
\left(g^{\prime}\right)^{n}=\tilde{q}(z) e^{P_{1}(z)}(g-a(z))^{n+1},
$$

where $\tilde{q}(z)=(-1)^{n}\left(\tau_{1}-z\right)^{n+1} q(z), a(z)=1 /\left(z-\tau_{1}\right)$. Write $G(z)=g(z)-a(z)$. Since $n \geq 2$, we have that $N(r, 1 / G)=N(r, 1 /(g-a))=S(r, g)$ by Lemma 3.3, (which holds when we consider $a(z)$ in place of $z$ ) and from (3.47),

$$
N\left(r, \frac{1}{G^{\prime}+a^{\prime}}\right)=N\left(r, \frac{1}{g^{\prime}}\right)=\frac{n}{n+1} N\left(r, \frac{1}{G}\right)+S(r, g)=S(r, g) .
$$

By Lemma 1.5,

$$
T(r, G) \leq 8 N\left(r, \frac{1}{G}\right)+8 N\left(r, \frac{1}{G^{\prime}+a^{\prime}}\right)+S(r, g) \leq S(r, g) .
$$

This yields a contradiction. For the proof of (i), we remark that (3.2) is a special case of (3.47). We have thus proved Proposition 3.4.

PROPOSITION 3.5. The equations (3.8) and (3.9) have no transcendental meromorphic solutions.

To prove Proposition 3.5, we need the lemma below.

LEMMA 3.6. Let $k(z) \not \equiv 0$ be a rational function and let $a_{1}(z), a_{2}(z), a_{3}(z)$ denote distinct rational functions. Suppose that a differential equation

$$
\left(-g^{\prime}+k(z) g^{2}\right)^{2}=q(z) e^{P_{1}(z)}\left(g-a_{1}(z)\right)\left(g-a_{2}(z)\right)\left(g-a_{3}(z)\right),
$$

possesses a transcendental meromorphic solution $g(z)$. Then $g(z)$ has infinitely many poles. 
ProOF. We assume that $g(z)$ has only finitely many poles and we shall find a contradiction. We differentiate both sides of (3.48). Combining this and (3.48) to eliminate $e^{P_{1}(z)}$, we get

$$
H\left(z, g, g^{\prime}\right) g^{4}+\Omega_{4}\left(z, g, g^{\prime}, g^{\prime \prime}\right)=0,
$$

with

$$
H\left(z, g, g^{\prime}\right)=\left(q(z) k(z) P_{1}^{\prime}(z)+q^{\prime}(z) k(z)-2 q(z) k^{\prime}(z)\right) g-q(z) k(z) g^{\prime},
$$

where $\Omega_{4}\left(z, g, g^{\prime}, g^{\prime \prime}\right)$ is a differential polynomial in $g$ of total degree at most 4 whose coefficients are rational functions. In view of the Clunie theorem we have $m(r, h)=S(r, g)$, where $h(z)=H\left(z, g(z), g^{\prime}(z)\right)$. Hence $h(z)$ is a small function with respect to $g(z)$ since we assume that $g(z)$ has only finitely many poles. For the simplicity, we write $g^{\prime}=\alpha(z) g+\beta(z)$ with small functions $\alpha(z)$ and $\beta(z)$. Substituting this into (3.48), we get

$$
q(z) e^{P_{1}(z)}=\frac{\left(k(z) g^{2}-\alpha(z) g-\beta(z)\right)^{2}}{\left(g-a_{1}(z)\right)\left(g-a_{2}(z)\right)\left(g-a_{3}(z)\right)} .
$$

This implies that there exist at least two distinct small functions $\gamma_{1}(z)$ and $\gamma_{2}(z)$ with respect to $g(z)$ such that

$$
N\left(f, \frac{1}{g-\gamma_{j}(z)}\right)=S(r, g), \quad j=1,2 .
$$

We apply Lemma 1.4 to $\infty, \gamma_{1}(z)$ and $\gamma_{2}(z)$ to obtain

$$
T(r, g) \leq \bar{N}(r, g)+\bar{N}\left(r, \frac{1}{g-\gamma_{1}}\right)+\bar{N}\left(r, \frac{1}{g-\gamma_{2}}\right)+S(r, g) \leq S(r, g),
$$

a contradiction. In fact, if the fraction in the right-hand side of (3.50) is irreducible, then $a_{j}(z) j=1,2,3$ can be chosen for $\gamma_{j}$ 's. This can be showed as follows: we fix $j$ among $j=1,2,3$ and suppose that $g-a_{j}(z)$ has infinitely many zeros more than small order, that is, $N\left(r, 1 /\left(g-a_{j}(z)\right)\right) \neq S(r, g)$. Write the numerator of the right-hand side of (3.50), $Q(z, g)$, as a polynomial in $g-a_{j}(z)$ :

$$
Q(z, g)=A_{j, 4}(z)\left(g-a_{j}(z)\right)^{4}+\cdots+A_{j, 1}(z)\left(g-a_{j}(z)\right)+A_{j, 0}(z),
$$

where $A_{j, k}, k=1, \ldots, 4$ are small functions. Let $z_{0}$ be a zero of $g-a_{j}(z)$. By (3.50), $z_{0}$ must be a zero of $A_{j, 0}(z)$ otherwise a pole of one of the other coefficients. Since $A_{j, k}(z)$ are small functions, $A_{j, 0}(z)$ must vanish, which means that $Q(z)$ is divided by $g-a_{j}(z)$. This argument implies that $N\left(r, 1 /\left(g-a_{j}\right)\right)=S(r, g)$, or that $Q(z, g)$ is divided by $g-a_{j}(z)$.

The right-hand side does not reduce to a polynomial, even though the right-hand side is reducible since $a_{j}(z)$ are distinct from each other. Thus we find $\gamma_{j}$ 's among $a_{j}(z), j=1,2,3$. 
ProOF OF Proposition 3.5. Suppose that the equation (3.8) has a transcendental meromorphic solution $f(z)$. By means of Lemma $3.3, f(z)$ has only finitely many fixed points. Put $g=1 /(f-z)$ in (3.8). Then we see that $g(z)$ satisfies a differential equation of the form (3.48). However, by Lemma $3.6, g(z)$ must have infinitely many poles, namely, $f(z)$ has infinitely many fixed points, a contradiction. Similarly we can show that (3.9) does not have any transcendental meromorphic solutions.

PROPOSITION 3.6. Suppose that equation (3.1) has a transcendental meromorphic solution $f(z)$. Then $s \leq 2$.

ProOF. We assume that $s \geq 3$ in (3.1), and show that this leads to a contradiction. Put $\varphi=1 /(f-z)$ in (3.1) to obtain

$$
\left(-\varphi^{\prime}+\varphi^{2}\right) \varphi^{s-2}=q(z) e^{P_{1}(z)} .
$$

We consider zeros and poles of $\varphi(z)$. By Lemma 3.2, $f(z)$ has only finitely many poles since $s \geq 3$. This implies that $\varphi(z)$ has only finitely many zeros. We divide the proof into two cases (I) when $\varphi(z)$ possesses only finitely many poles, and (II) when $\varphi(z)$ possesses infinitely many poles.

(I). In this case, we see that $F(z):=f(z)-z$ has only finitely many zeros. It follows from this and (3.1) that $F^{\prime}(z)+1$ has only finitely many zeros. We make use of Lemma 1.5 to get

$$
T(r, F) \leq 8 N\left(r, \frac{1}{F}\right)+8 N\left(r, \frac{1}{F^{\prime}+1}\right)+S(r, F) \leq S(r, F),
$$

a contradiction.

(II). Let $z_{0}$ denote a pole of $\varphi(z)$ satisfying $q\left(z_{0}\right) \neq 0, \infty$. It follows from (3.51) that $\Phi(z):=-\varphi^{\prime}(z)+\varphi(z)^{2}$ must have a zero at $z_{0}$, which implies that $\varphi(z)$ has a simple pole with residue -1 . We write $\varphi(z)$ in a neighbourhood of $z_{0}$ as

$$
\varphi(z)=\frac{-1}{z-z_{0}}+c_{0}+c_{1}\left(z-z_{0}\right)+O\left(z-z_{0}\right)^{2} .
$$

This gives that in a neighbourhood of $z_{0}$

$$
\begin{aligned}
\Phi(z)= & -\left(\frac{1}{\left(z-z_{0}\right)^{2}}+c_{1}+O\left(z-z_{0}\right)\right) \\
& +\left(\frac{1}{\left(z-z_{0}\right)^{2}}-\frac{2 c_{0}}{z-z_{0}}+c_{0}^{2}-2 c_{1}+O\left(z-z_{0}\right)\right) \\
= & \frac{-2 c_{0}}{z-z_{0}}+c_{0}^{2}-3 c_{1}+O\left(z-z_{0}\right) .
\end{aligned}
$$


Since $\Phi(z)$ admits zero at $z_{0}$, we see that $c_{0}=c_{1}=0$. Namely,

$$
\varphi(z)=\frac{-1}{z-z_{0}}+O\left(z-z_{0}\right)^{2} .
$$

Using this, we have that

$$
\frac{\varphi^{\prime}(z)}{\varphi(z)}=\frac{-1}{z-z_{0}}+O\left(z-z_{0}\right)^{2} .
$$

It follows from (3.53) that a meromorphic function

$$
b(z):=\left(\frac{\varphi^{\prime}(z)}{\varphi(z)}\right)^{2}-\left(\frac{\varphi^{\prime}(z)}{\varphi(z)}\right)^{\prime}=\varphi\left(\frac{1}{\varphi}\right)^{\prime \prime}
$$

has a zero at $z_{0}$. Then we have

$$
N(r, b)=O(\log r)
$$

and if $b(z) \not \equiv 0$, then

$$
N(r, \varphi) \leq N\left(r, \frac{1}{b}\right)+S(r, \varphi) \leq T(r, b)+O(\log r) .
$$

By the theorem on the logarithmic derivatives, we get

$$
m(r, b) \leq 3 m\left(r, \frac{\varphi^{\prime}(z)}{\varphi(z)}\right)+O(\log r) \leq S(r, \varphi) .
$$

Combining (3.55) and (3.57), we see that $T(r, b)=S(r, \varphi)$. From (3.56) we have

$$
N\left(r, \frac{1}{f-z}\right)=N(r, \varphi)=S(r, \varphi)=S(r, f) .
$$

By (3.1) we have

$$
N\left(r, \frac{1}{f^{\prime}}\right)=s N\left(r, \frac{1}{f-z}\right)+O(\log r)=S(r, f) .
$$

Again applying Lemma 1.5 to $F(z)=f(z)-z$, we obtain

$$
T(r, F) \leq 8 N\left(r, \frac{1}{F}\right)+8 N\left(r, \frac{1}{F^{\prime}+1}\right)+S(r, F) \leq S(r, F),
$$

a contradiction. Hence $b(z)$ must vanish. However, by (3.54), $\varphi(z) \equiv 0$, or $1 / \varphi(z)$ is a linear polynomial or a constant, a contradiction. Thus we have proved Proposition 3.6.

PROOF OF THEOREM 1.2. In view of Lemma 3.1, we may treat the equations (3.1)(3.9) separately. By Proposition 3.5, the equations (3.8) and (3.9) have no transcendental meromorphic solutions. By Proposition 3.2, the equation (3.7) must be of the 
form (1.11). By Proposition 3.3 (i), the equation (3.3) must be of the form (1.9) or (1.10). By Proposition 3.3 (ii), the equation (3.6) must be of the form (1.10) or (1.11). By Proposition 3.4 (i)-(ii), the equations (3.2) and (3.5) should be of the form (1.8). By Proposition 3.6, the equation (3.1) must be an equation of the form (1.7) or (1.8). We finally consider the equation (3.4). By Proposition 3.1, it must be an equation of the form (3.1) or (3.3). Hence we conclude that the equation (3.4) must be one of the equations of the form $(1.7),(1.8),(1.9)$ or $(1.10)$. Therefore, we have proved Theorem 1.2.

\section{Acknowledgements}

The authors would like to thank the referee for valuable suggestions. They would also like to thank Professor Ilpo Laine for his helpful discussions and useful advice.

\section{References}

[1] S. B. Bank and R. P. Kaufman, 'On the meromorphic solutions of first-order differential equations', Comment. Math. Helv. 51 (1976), 289-299.

[2] - 'On the growth of meromorphic solutions of the differential equation $\left(y^{\prime}\right)^{m}=R(z, y)$ ', Acta Math. 144 (1980), 223-248.

[3] S. B. Bank, G. G. Gundersen and I. Laine, 'Meromorphic solutions of the Riccati differential equation', Ann. Acad. Sci. Fenn. Ser. A I Math. 6 (1981), 369-398.

[4] A. Beardon, Iteration of rational functions (Springer, New York, 1991).

[5] W. Bergweiler, 'Newton's method and a class of meromorphic functions without wandering domains', Ergodic Theory Dynamical Systems 13 (1993), 231-247.

[6] _ - 'Iteration of meromorphic functions', Bull. Amer. Math. Soc. 29 (1993), 151-188.

[7] — ' 'On a theorem of Gol'dberg concerning meromorphic solutions of algebraic differential equations', Complex Variables Theory Appl. 37 (1998), 93-96.

[8] W. Bergweiler and N. Terglane, 'Weakly repelling fixpoints and the connectivity of wandering domains', Trans. Amer. Math. Soc. 348 (1996), 1-12.

[9] W. Bergweiler and N. Terglane, 'On the zeros of solutions of linear differential equations of the second order', J. London Math. Soc. (2) 58 (1998), 311-330.

[10] A. A. Gol'dberg, 'On single-valued solutions of first order differential equations' (Russian), Ukrain. Math. Zh. 8 (1956), 254-261.

[11] W. K. Hayman, Meromorphic functions (Clarendon Press, Oxford, 1964).

[12] W. K. Hayman and J. Miles, 'On the growth of a meromorphic functions and its derivatives', Complex Variables Theory Appl. 12 (1989), 245-260.

[13] Y. He and I. Laine, 'The Hayman-miles theorem and the differential equation $\left(y^{\prime}\right)^{n}=R(z, y)$ ', Analysis 10 (1990), 387-396.

[14] E. Hille, Ordinary differential equation in the complex domain (Wiley and Sons, New York, 1976).

[15] G. Jank and L. Volkmann, Meromorphe Functionen und Differentialgleichungen (Birkhäuser, Basel, 1985).

[16] M. Jankowski, 'Newton's method for solutions of quasi-Bessel differential equations', Ann. Acad. Sci. Fenn., Math. 22 (1997), 187-204. 
[17] I. Laine, Nevanlinna theory and complex differential equations (W. Gruyter, Berlin, 1992).

[18] A. Z. Mokhon'ko and V. D. Mokhon'ko, 'Estimates for the Nevanlinna characteristic of some classes of meromorphic functions and their applications to differential equations', Sib. Math. J. 15 (1974), 921-934.

[19] R. Nevanlinna, Analytic functions (Springer, Berlin, 1970).

[20] N. Steinmetz, Eigenschaften eindeutiger Lösungen gewöhnlicher Differentialgleichungen im Komplexen (Ph. D. Thesis, Karlsruhe, 1978).

[21] — 'Meromorphe Lösungen der Differentialgleichungen $Q(z, w)\left(d^{2} w / d z^{2}\right)^{2}=$ $P(z, w)(d w / d z)^{2}$, Complex Variables Theory Appl. $10(1988), 31-41$.

[22] — Rational iteration (W. Gruyter, Berlin, 1993).

[23] Y. Wang, 'Wandering domains in the dynamics of certain meromorphic functions', Bull. Austr. Math. Soc. 59 (1999), 99-106.

[24] K. Yosida, 'A generalization of Malmquist's theorem', Japan J. Math. 9 (1933), 253-256.

[25] J. H. Zhu, 'The general form of Hayman's inequality and the fixed points of meromorphic functions', Chinese Sci. Bull. 33 (4) (1988), 265-269.

Department of Mathematics

Nippon Institute of Technology

4-1 Gakuendai Miyashiro

Minamisaitama Saitama 345-8501

Japan

e-mail: ishi@nit.ac.jp
Institute of Mathematics, AMSS

Chinese Academy of Sciences

Beijing 100080

China

e-mail: wangyf@math03.math.ac.cn 